\title{
The Delaunay/Voronoi Selection Graph: a Method for Extracting Shape Information from 2-D Dot-Patterns with an Extension to 3-D.
}

\author{
Glynn Robinson, Lewis Griffin \& Alan Colchester. \\ Department of Neurology, \\ Guy's Hospital, London, England, SE1 9RT.
}

\begin{abstract}
In this paper we present the Delaunay/Voronoi selection graph (DVSG), an approach to the representation of the shape of 2-D objects which does not require a complete segmentation, merely a pattern of dots which are believed to lie on the edges of objects. The technique produces both a skeleton and boundary representation and, with subsequent processing, generates a hierarchical description of the topology of individual objects. We compare the DVSG to other methods used for obtaining information from dot-patterns, and show how this technique can be extended to 3-D.
\end{abstract}

\section{Introduction}

We require a method of representing the shapes of objects following an initial segmentation of an image. Since segmentation techniques often fail to produce a complete segmentation, we chose to treat candidate edge points as individual dots and compute both a skeleton and boundary representation for the perceived objects within the dot pattern. The method of shape representation we have developed is based on the Delaunay triangulation and its dual the Voronoi diagram of a set of points [1]. We refer to the method as the Delaunay/Voronoi selection graph (DVSG). Subsequent processing produces a hierarchical representation both of the boundary and the skeleton of objects. The hierarchical structure reduces its sensitivity to small changes along the object boundary and also facilitates coarse to fine matching of image features to model entities.

Many approaches to shape representation have been proposed, and more extensive reviews can be found in references [2,3]. Boundary representations of the shape of objects such as those described in references $[4,5]$ tend to be sensitive to small changes along the object boundaries, and hierarchical representation is often difficult, as is the sub-division of objects into their sub-parts. The hierarchical approach of the curvature primal sketch [6] uvercomes some of the probienis of boundary representations, but the use of multiple Gaussian scales may cause problems especially when primitives are close together.

Skeleton representations such as proposed in $[5,7]$ allow objects to be represented in terms of the relationships between their sub-parts. Spurious skeleton branches can be generated by small protrusions on the object boundary. 
Nackman \& Pizer [8] propose an approach which overcomes the problems of these spurious branches by generating the skeleton of an object at multiple Gaussian scales, and Arcelli [9] proposes a hierarchy of skeletons in terms of the object's boundary curvature.

The problem of grouping together dots to form perceptual groups has been attempted by a number of authors. Fairfield $[10,11]$, like ourselves, is concerned with both the detection of the boundary of objects from dots, and also the segmenting of these objects into their sub-parts. He uses the Voronoi diagram to detect areas of internal concavity and replacing Voronoi diagram sides with the corresponding Delaunay triangulation side to produce both the object boundary and sub-parts. This work is dependent on a user defined threshold and does not differentiate between object boundaries and the sub-part boundaries. Ogniewicz et. al. [12] use the Voronoi diagram of a set of points to produce a medial axis description of objects. This method requires that the points making up the boundary have known connectivity, and a threshold is used to prune the skeleton description. Ahuja et. al. [13,14] propose the use of the Voronoi diagram and the properties of the individual Voronoi cells to classify points as boundary points, interior points, isolated points, or points on a curve.

Perceptual grouping algorithms based on the Delaunay Triangulation (DT) have been proposed by several other authors $[15,16]$. Three subgraphs of the DT which are of particular interest are: the Gabriel Graph (GG) [17]; the Relative Neighbourhood Graph (RNG) [18]; the Minimum Spanning Tree (MST). These graphs are shown in Figure 3 and defined below.

The Gabriel Graph is defined as follows: any edge $<\mathrm{u}, \mathrm{v}\rangle$ of the DT is an edge of the GG iff the circle with $\langle\mathrm{u}, \mathrm{v}\rangle$ as diameter contains no points in its interior. The Relative Neighbourhood Graph is defined as follows: any edge $\langle\mathrm{u}, \mathrm{v}\rangle$ of the DT is an edge of the RNG iff the lune formed by the intersection of the circles centred at $\mathrm{u}$ and $\mathrm{v}$ with radius $|\langle\mathrm{u}, \mathrm{v}\rangle|$ contains no points in its interior. The Minimum Spanning Tree is the tree of minimal total length which visits every point. Section 3 compares these three graphs to the graph generated by our technique.

Our method, the DVSG, does not require connected boundaries as input, merely a set of points (dots) which are believed to be edge points of objects. It produces distinct objects from these potential edge points, and concurrently generates both a skeleton and boundary representation of the shape of these objects.

\section{Methods}

\subsection{Selecting skeleton and boundary sections.}

Input to our technique comes in the form of disconnected dots. Each dot is assumed to lie on the edge of an unspecified object(s). We assume that the sides in the Delaunay triangulation provide a superset of the boundaries of the perceived objects within the dot-pattern. Likewise, we assume that the sides of the Voronoi diagram form a superset of the skeletons of the perceived objects.

In our approach, the problem of boundary and skeleton definition is simplified to selecting from the Voronoi diagram those sides that form the object 
skeleton, and selecting from the Delaunay triangulation those sides that form the object boundary. An example is shown in figure 1 . We observe that when a Delaunay side is perceived to lie on the boundary (e.g. $A B$ in figure $1 b$ ), the Voronoi side corresponding to this Delaunay side is NOT perceived to be on the skeleton (e.g. CD in figure 1b). Further, when a Voronoi side is perceived to lie on the skeleton, the corresponding Delaunay side is NOT perceived to lie on the boundary. The problem is thus further refined into a simple choice between a Delaunay side and its corresponding Voronoi side. We make this choice by keeping the shorter of the Delaunay side or its associated Voronoi side. If the Delaunay side is shorter then this is added to the list of boundary sections; if on the other hand the Voronoi side is shorter, this is added to the list of skeleton sections. This can be viewed as deciding whether any two points connected by the Delaunay triangulation are to be regarded as adjacent points on the same boundary, in which case we choose to keep the Delaunay side connecting them (e.g. $\mathrm{AB}$ in figure $1 \mathrm{~b}$ ); or whether they are lying opposite each other and separated by a skeleton, in which case we choose to keep the associated Voronoi section as part of the skeleton (e.g. CE in figure 1b). We call the graph resulting from this selection process the Delaunay/Voronoi Selection Graph (DVSG). Figure 2a shows an example image, and figure $2 \mathrm{~b}$ shows a series of points outlining the major features within the image. Figure $2 \mathrm{c}$ shows the result of the above selection criterion for the points in figure $2 \mathrm{~b}$ (skeleton sections are shown as dashed lines; boundary sections as solid lines).

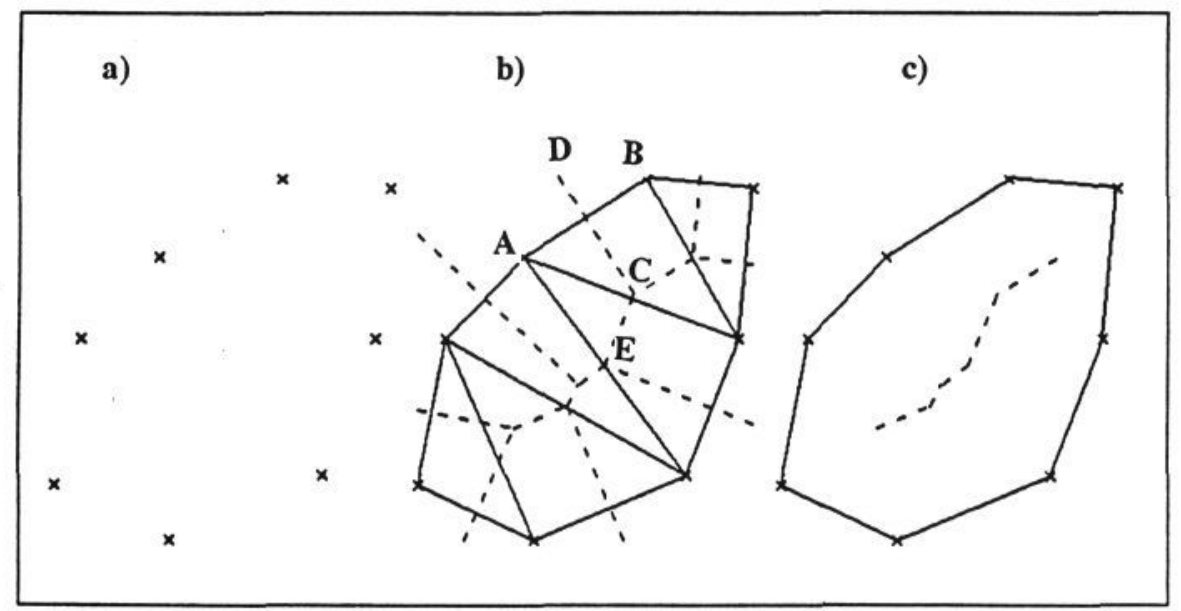

Figure 1 a) sample input points; b) Delaunay triangulation (solid) and Voronoi Diagram (dashed) c) result of the selection process.

\subsection{Defining objects}

Since no prior knowledge about the connectivity between edge points is necessarily provided, and there is no information about the number of objects present, it is necessary to define the objects that have been created following the creation of the DVSG. Following the initial selection of boundary and skeleton sections, sequences of connected skeleton sections (which may posses multiple branches) exist. These 
connected skeleton sections are surrounded by boundary sections. We define an object as a sequence of connected skeleton branches and the surrounding boundary sections. Each pair of points which was connected in the original Delaunay triangulation is now classed as either defining a boundary side or a skeleton section, and is also associated with one object (if the pair defines a skeleton section) or one or two objects (if the pair defines a boundary side). Figure 2d shows the computed boundary for the largest object in figure $2 \mathrm{c}$.

\subsection{Splitting objects into sub-parts}

Each branching object is next segmented into its sub-parts. This is accomplished in the following way. All skeleton branches are three way and occur where all three Voronoi sides corresponding to one Delaunay triangle were shorter than their respective triangle sides. Each branch point in an object's skeleton is considered as either: 1) the meeting point of a small part of the object with the larger, main body of the object. The smaller part is considered as less important than the main body and is broken off as a sub-part; 2) the meeting point of two smaller sections with the main body of the object. Both of the smaller sections are then broken off as subparts.

Associated with each branch we define an area and a direction. The area is the sum of the areas of all of the triangles along that branch. The direction is the direction of the line from the branch point through the centre of gravity of the mid-points of the skeleton sections making up that branch. The centre of gravity is calculated from the mid-points of each Voronoi section in the skeleton of that branch, and each mid-point is weighted by the length of its skeleton section. The allows longer skeleton sections to have more effect on the direction of the branch than smaller ones.

For 1) above to be chosen, the branch with the smallest area must be significantly different from the branch with the second smallest area. This measure of significance is defined by the ratio of the two areas. Branch "a" is significantly smaller than branch " $b$ " if area(a)/area(b) $<0.2$. The branch with the smallest area is then broken off. Failing this, the sine of the difference between the direction of the smallest and the largest branch is compared to the sine of difference between the direction of the second smallest branch and the largest branch. The sine is taken as this gives us a large value if the directions are similar and a small value if the directions are dissimilar. If the ratio of the smaller of these two values over the larger falls below 0.2 we assume that one branch has a direction significantly different to that of the largest branch, and we break off the branch with a direction most different to that of the largest branch.

If neither of the above two conditions are met we assume that 2) above is true, and we break off both of the smaller branches.

When a branch is classified as belonging to a sub-part, its Voronoi section emanating from the branch is flagged as a cut stem. All of the skeleton and boundary sections of that sub-part are then labelled accordingly. The Delaunay sides corresponding to the skeleton cut stems form the virtual boundaries between sub-parts. This is an example of how the duality between the Voronoi diagram and the Delaunay triangulation makes it very easy to add/remove sections of an object by simply toggling a triangle side. Figure $2 \mathrm{e}$ shows the part boundaries (dotted 
lines) for the object of figure $2 \mathrm{~d}$.

\subsection{Generating the intra object hierarchy}

The sub-parts of each object are next represented hierarchically as a coarse-to-fine description. For each object, the sub-parts are ordered in terms of decreasing area. A level in the hierarchy is placed wherever there is a large change in area between successive sub-parts in this ordered list. Currently this is accomplished by looking for maxima in the derivative of the list. By successively adding the different levels of the hierarchy to the object description a more detailed version of the object can be obtained. Unlike many other approaches to hierarchial, coarse-to-fine descriptions, the position of the boundaries does not change as we go from coarse to fine; it is simply the case that some virtual boundaries are replaced either by virtual boundaries at a more detailed level of description, or by the final boundary of the object. Figure 2f-h show the different levels of the hierarchy for the object of figure $2 \mathrm{~d}$. The finest detail is shown in figure $2 \mathrm{~h}$.

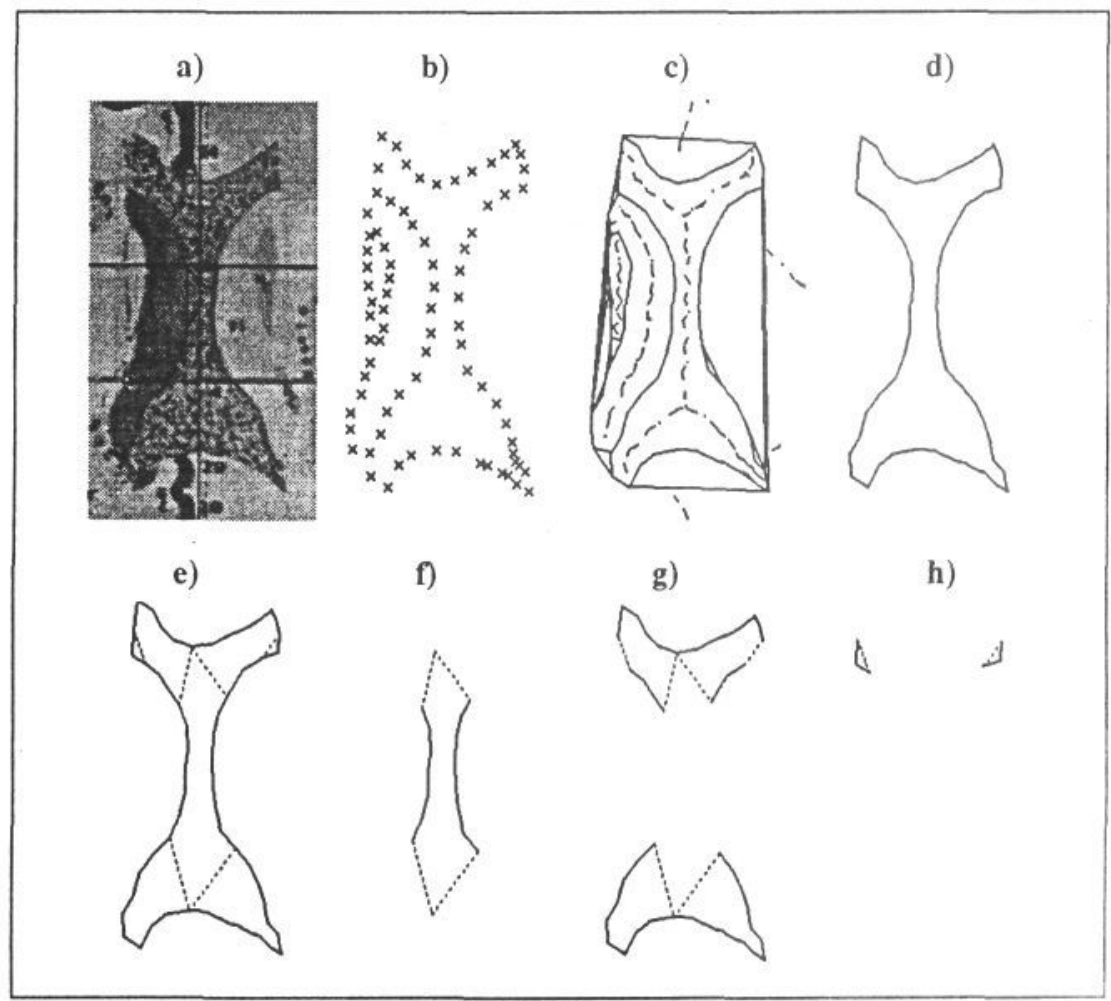

Figure 2 a) Original image; b) input points; c) Result of selection; d) Major object; e) parts of major object; f-h) hierarchy for major object. 


\section{Comparison with other subgraphs of the Delaunay triangulation}

This section compares the DVSG with the GG, RNG and MST (defined in section 1). Figure 3 shows an example of these graphs. Several points can be made from comparing these graphs:

1. It is well known that the relation DT $\supset$ GG $\supset$ RNG $\supset$ MST holds [19]. Inspection of figure 3 . shows that the DVSG does not fit into this sequence and in fact no inclusion relationship exists between the DVSG and the GG, RNG or MST.

2. The GG, RNG and MST are necessarily connected whilst the DVSG is not. 3. The DVSG always contains the convex hull whilst the GG, RNG and MST do not.

This lack of relation between the DVSG and the GG, RNG and MST can be understood by appreciating that the DVSG was developed with the aim of connecting points into coherent boundaries, whereas the other graphs have had there main application in clustering points into dense groups.

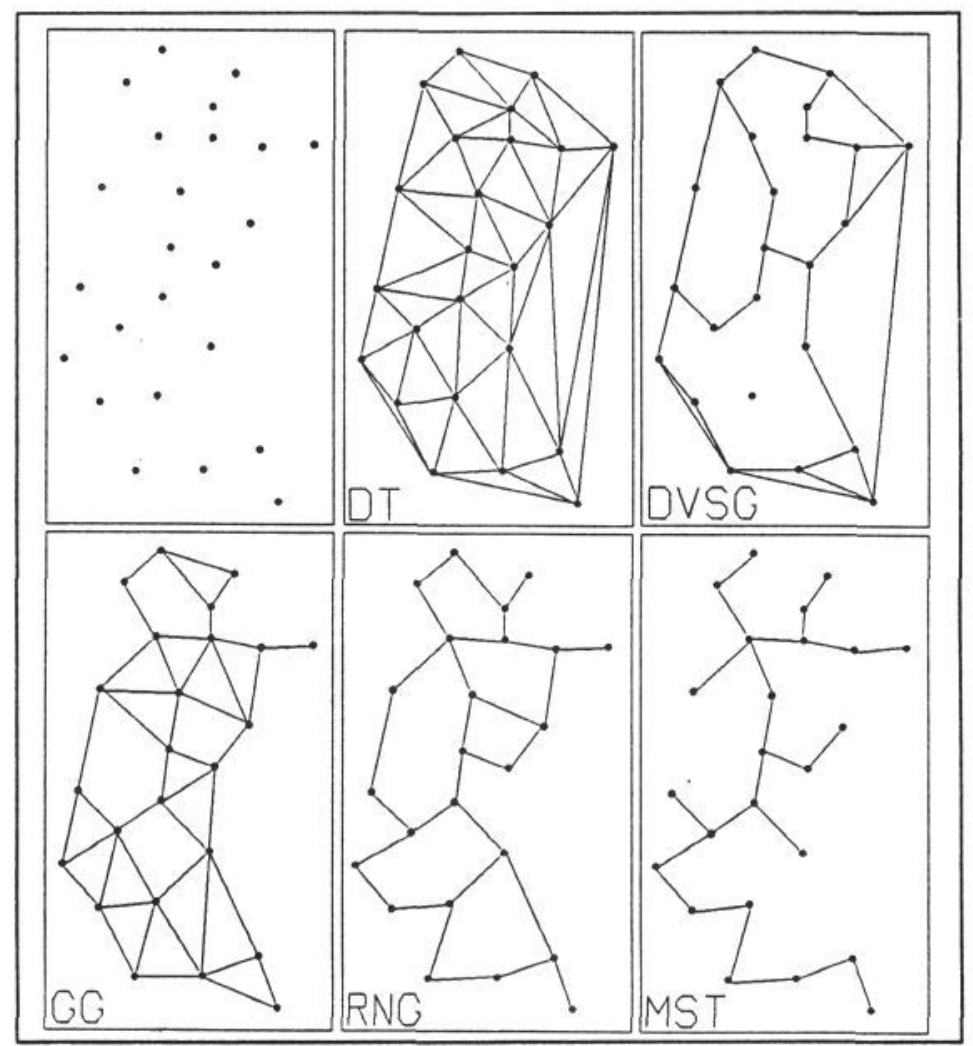

Figure 3 Comparison of the DVSG with subgraphs of the DT. 


\section{Extension to 3-D}

The 2-D Delaunay triangulation has a 3-D equivalent. In 3-D points are connected into tetrahedra (c.f. triangles in 2-D). Each tetrahedron has no points in the interior of its circumsphere. The Voronoi diagram is also extendable to 3-D. Lines connecting the circumsphere centres of adjacent tetrahedra form Voronoi sides. Each Voronoi cell is the volume around a point closer to that point than any other.

Each face of a Delaunay tetrahedron has associated with it one side from the 3-D Voronoi diagram. Likewise, each face of the 3-D Voronoi diagram has associated with it one side of a Delaunay tetrahedron.

As in the 2-D case, input points to the 3-D extension are assumed to be potential edge points of 3-D objects.

Each face of a Delaunay tetrahedron is assumed to be a potential surface facet of an object. The decision that needs to be made for every face is whether it should be kept as a surface facet (when its points lie close to each other) or whether it should be ignored and the corresponding Voronoi side kept as a 3-D skeleton section. The method used here compares the absolute length of the Voronoi side to the mean length of the three sides making up the Delaunay face to which it corresponds. If the mean length of the Delaunay sides is less than the Voronoi side then the face is retained as a surface facet. Otherwise the Voronoi side is retained as a skeleton section.

The surface facets are individual Delaunay faces. Had the selection process been based around individual Delaunay sides then it would have been possible to generate a single one dimensional line as an individual surface. This does not seem desirable, especially when assuming that the tetrahedron faces form a superset of the object surface.

Skeletons can take a number of forms. At their simplest they can be single points corresponding to the circumsphere centre of a tetrahedra which has had all four of its faces retained as surface. More complex skeleton sections can be created. These more complex sections comprise 1) skeleton lines which are stretches of one dimensional polylines connecting the centres of circumspheres of adjacent tetrahedra; 2) skeleton sheets which arise when all of the faces that a particular Delaunay side is an edge of are retained as skeleton sections. Sheets occur when the surface generated cannot be described by a one dimensional axis. Figure 4a shows two tetrahedra formed from five points. Figure $4 \mathrm{~b}$ shows the creation of a skeleton line which replaces a single tetrahedron face. Figure 5a shows four tetrahedra formed from six points. Figure 5b shows a skeleton sheet formed by replacing all of the tetrahedron faces of which the Delaunay triangle side $A B$ is a part with Voronoi sections.

Objects are defined in the same way as for the 2-D case. They are sequences of unbroken (and possibly branching) skeleton sections (normal and sheets), and surrounding Delaunay faces. 
a)

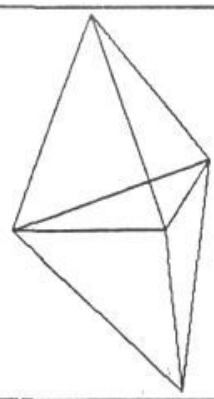

b)

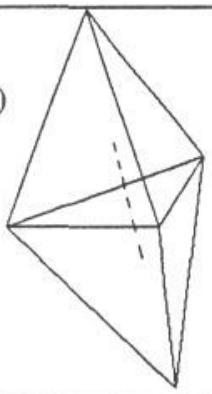

Figure 4 a) Two tetrahedra; b) tetrahedra and skeleton section (dashed).

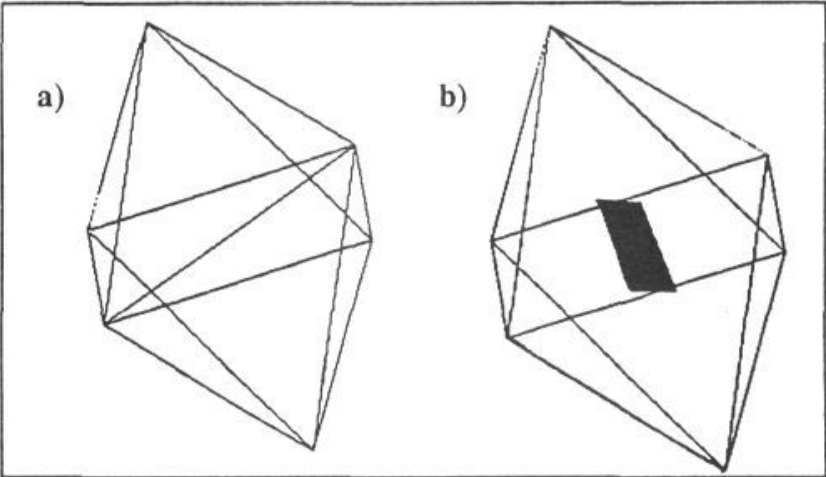

Figure 5 a) four tetrahedra; b) tetrahedra and skeleton sheet (solid)

\section{Discussion}

We construct a shape description of each object by decomposing the object into a hierarchy of sub-parts. Our technique simultaneously extracts boundary and skeleton representations of multiple objects from incomplete edge data. The input candidate edge points require no connectivity information. Objects are defined simultaneously in terms of their intact skeleton and boundary.

The connections between the points are generated through the algorithm using local geometry only, but a simple modification allows the user to specify partial connectivity or complete connectivity between points. Using information about connectivity means that the technique can be used as a simple edge connection algorithm [20] The purely local decision could be improved by incorporating information about continuity into the algorithm.

The creation of the skeleton and boundary sections for objects is a separate process from that of segmenting the objects into sub-parts and generating the hierarchical representation. It should be noted that the technique proposed here for generation of the skeleton and boundary sections can be used in conjunction with any other algorithm for segmenting the skeleton; and also that the technique proposed here for segmenting the skeleton could be used with any other method for generating sub-parts and hierarchies.

The current method of segmenting individual objects into their sub-parts 
usually appears to correspond well to subdivisions perceived by observers. This implies that the criteria of size and direction are sensible ones to use. However there are occasions where a different segmentation would be desirable. This is usually where a skeleton has no branch but the boundary that it separates has a significant narrowing. Use of the rate of change of the boundary-to-skeleton distance could be included as an extra criterion when deciding where to segment objects into sub-parts. We currently use an empirically determined threshold for deciding whether to split an object into sub-parts. The threshold is a ratio of local values (in the feature space) and is relatively insensitive to small changes in these values. However, a more robust approach is required.

The use of minima in the derivative of the ordered list of sub-parts provides a generally good hierarchical description. The major problem with the current approach is that there are superfluous levels in the hierarchy towards the fine end description, since the levels only use local criteria, and small perturbations in the size of the sub-parts means there are a large number of minima in the first differential. Alternative criteria for the hierarchical ordering of sub-parts could be used such as large percentage change in the size of sub-parts.

Our technique provides an easy method for splitting one object into two or combining two objects into one by simply changing the labelling of the Voronoi section and its corresponding Delaunay section [21]. This splitting and merging could be controlled by high-level knowledge in an attempt to match features in a model to objects generated by the proposed shape description. We are currently undertaking work in defining efficient metrics with which to match object descriptions extracted from the input data to those held within the high level model. In conjunction with this, we are extending our high level model[22] such that all features have a shape representation in the same form as the one described in this paper.

The technique can also be extended to 3-D. We have shown preliminary results of an extension to $3-\mathrm{D}$ using a proximity measure computed from the mean length of the sides of a Delaunay tetrahedron face and the length of the associated Voronoi side.

\section{References}

[1] D. T. Lee and B. J. Schacter, Two algorithms for constructing a Delaunay triangulation. Int J Comp Info Sci 1980; 9 No. 3: 219-242.

[2] D. R. Bailes and D. H. Cooper, A Literature Review of Representation of Two Dimensional Shape, Uni. Manchester Tech. Report Mobprim/Mu/Lr1/881209, 1988. (UnPub)

[3] S. Marshall, Review of Shape Coding Techniques. Image and Vision Computing 1989; 7 No. 4: 281-294.

[4] H. Freeman, On the encoding of arbitary geometric configurations. IRE Trans Electronic Computers 1961; June: 260-268.

[5] N. J. Ayache, A model-based vision system to identify and locate partially visible industrial parts. Proceedings of the IEEE Computer Society Conference on Computer Vision and Pattern Recognition. IEEE, New York, 1983, pp 492-494.

[6] H. Asada and M. Brady, The Curvature Primal Sketch. IEEE Trans Pat 
Anal Machine Intel 1986; PAMI-8 N0. 1: 2-14.

[7] H. Blum, Biological Shape and Visual Science. J Theor Biol 1973; 38: 205-287.

[8] L. R. Nackman and S. M. Pizer, Three dimensional shape description using the symmetric axis transform. IEEE Trans Pat Anal Machine Intel 1985; PAMI-9 No. 4: 505-511.

[9] C. Arcelli, Pattern thinning by contour tracing. Comput Graphics Image Process 1981; 17: 130-144.

[10] J. R. C. Fairfield, Segmenting blobs into subregions. IEEE Trans Sys Man Cyb 1983; SMC-13 No. 3: 363-384.

[11] J. R. C. Fairfield, Segmenting dot patterns by Voronoi diagram concavity. IEEE Trans Pat Anal Machine Intel 1983; PAMI-5 No. 1: 104-110.

[12] R. Ogniewicz and M. Ilg, Skeletons with euclidean metric and correct topology and their application in object recognition and document analysis. Proceedings 4th International Symposium on Spatial Data Handling. Zurich, Switzerland, 1990, pp 15-24.

[13] N. Ahuja, Dot Pattern Processing Using Voronoi Neighbourhoods. IEEE Trans Pat Anal Machine Intel 1982; PAMI-4 No. 3.: 336-343.

[14] N. Ahuja and M. Tuceryan, Extraction of Early Perceptual Structure in Dot Patterns: Integrating region, boundary \& component Gestalt. Comp Vis Graph Image Proc 1989; 48: 304-346.

[15] F. Meyer, The perceptual graph: a new algorithm. Proc. Int. Congr. Acoust. Speech Signal Process. Paris, 1982,

[16] G. T. Toussaint, The relative neighbour graph of a finite planar set. Patt Recog 1980; 12(4): 1324-1347.

[17] K. R. Gabriel and R. R. Sokal, A new statistical approach to geographic variations analysis. Systematic Zoology 1969; 18: 259-278.

[18] P. M. Lankford, Regionalisation: theory and alternative algorithms. Geograph Anal 1992; 1: 196-212.

[19] L. Vincent, Graphs and mathematical morphology. Signal Processing 1989; 16: 365-388.

[20] G. P. Robinson, A. C. F. Colchester and L. D. Griffin, A hierarchical shape representation for use in anatomical object recognition. In: Achcrya, R. S., Cogswell, C. J. and Goldof, D. B. (ed) Proc. SPIE Biomedical Image processing III and 3-D Microscopy. San Jose, 1992, pp 594-605.

[21] G. P. Robinson, A. C. F. Colchester, L. D. Griffin, et al., Integrated skeleton and boundary shape representation for medical image interpretation. In: Sandini, G. (ed) Proc. European Conference Computer Vision. LNCS-Series Vol 588. Springer-Verlag, 1992, pp 725-729.

[22] C. I. Attwood, G. D. Sullivan, G. P. Robinson, et al., Model-based interpretation of anatomical structures in cranial MR images. Proc. British Machine Vision Conference. Sheffield University Press, 1990, pp 145-150. 